\title{
Thermal exfoliation of electrochemically synthesized graphite intercalation compound with perrhenic acid
}

\author{
Bartosz Gurzęda $^{1}$ (D) $\cdot$ Tomasz Buchwald $^{2}$ (i) $\cdot$ Piotr Krawczyk $^{1}$ (i)
}

Received: 9 April 2020 /Revised: 5 May 2020 / Accepted: 7 May 2020 / Published online: 16 May 2020

(C) The Author(s) 2020

\begin{abstract}
In present work, we describe the synthesis of graphite intercalation compounds with perrhenic acid ( $\mathrm{HReO}_{4}$-GIC) through the anodic oxidation of graphite in aqueous perrhenic acid solution and their thermal exfoliation. Due to electrochemical treatment of graphite in perrhenic acid solution, $\mathrm{ReO}_{4}^{-}$ions are intercalated into interlayer spaces of graphite. Anodic oxidation of graphite in $\mathrm{HReO}_{4}$ solution leads to the formation of 3-stage GIC. Simultaneously, some amount of perrhenic acid becomes deposited on the graphite surface and edges. In the next step, thermal treatment of the previously synthesized GIC was performed, causing both the exfoliation of graphitic structure and transformation of perrhenic acid into rhenium oxides on the surface of graphene layers. The yielded product was exfoliated graphite- $\mathrm{ReO}_{2} / \mathrm{ReO}_{3}$ composite. The obtained composite was characterized by X-ray diffraction (XRD), scanning electron microscopy (SEM), and Raman spectroscopy. Additionally, specific surface area of the exfoliated materials was measured.
\end{abstract}

Keywords Graphite intercalation compound $\cdot$ Perrhenic acid $\cdot$ Electrochemical oxidation $\cdot$ Exfoliation $\cdot$ Rhenium oxide

\section{Introduction}

Due to the presence of relatively weak van der Waals forces between the graphene layers in graphite, ions, molecules, or atoms can be inserted into the interlayer spaces of graphite matrix. This process is called intercalation and the yielded product is graphite intercalation compound (GIC) $[1,2]$. The direction of electron transfer between the graphite matrix and intercalate defines the type of synthesized GIC. When intercalate accepts electrical charge from the carbon matrix, an acceptor GIC is synthesized. Contrariwise, if the electrical charge is accepted by carbon matrix, the donor-type GIC is obtained [3]. Most of donor-type intercalates are alkali metals, i.e., $\mathrm{Li}, \mathrm{Na}, \mathrm{K}$, or ammonium quaternary salts [4]. On the other hand, acceptor GICs commonly contain inorganic acids, i.e., $\mathrm{H}_{2} \mathrm{SO}_{4}, \mathrm{HNO}_{3}, \mathrm{HClO}_{4}[5,6]$, or metal chlorides [7, 8]. The efficiency of intercalation process is specified by the

Bartosz Gurzęda

bartosz.gurzeda@put.poznan.pl

1 Institute of Chemistry and Technical Electrochemistry, Poznan University of Technology, Berdychowo 4, 60965 Poznan, Poland

2 Institute of Materials Research and Quantum Engineering, Poznan University of Technology, Piotrowo 3, 60965 Poznan, Poland intercalation stage. It reveals the number of graphene layers separated by the neighboring layers of intercalate, for example, if every intercalant layer is separated by two graphene layers, the pure 2-stage GIC is synthesized $[2,3]$.

GIC can be synthesized on the way of chemical $[9,10]$, electrochemical [11-13], or photochemical reaction [14]. Chemical methods are divided into these carried out in gas or liquid phase. In the first one, the vapors of intercalate are passed through the bed of graphite, i.e., $\mathrm{N}_{2} \mathrm{O}_{5}, \mathrm{SO}_{3}, \mathrm{Br}_{2}$, or $\mathrm{Re}_{2} \mathrm{O}_{7}$ [15-18], whereas an intercalation in liquid phase is realized in molten salts, i.e., $\mathrm{ZnCl}_{2}, \mathrm{NiCl}_{2}$, or $\mathrm{FeCl}_{3}$, or in the dissolved intercalate $[19,20]$. However, the commonly used system comprises of inorganic acids, such as sulfuric or perchloric, dissolved in water $[5,10]$. To start the chemical intercalation, the addition of reductant or oxidant, depending on the type of prepared GIC, is needed. Electrochemical intercalation is carried out by polarization of graphite in dissolved intercalate, which plays a role of electrolyte. Depending on the electrons flow direction, the electrochemical processes are performed by anodic oxidation or cathodic reduction. Contrary to chemical methods, the electrochemical ones are more controllable and the product with desired properties is easily obtained. Moreover, they are environmental friendly, because no oxidant/reductant agent is used, and the final product has no impurities caused by the presence of 
oxidation/reduction by-products [21]. On the other hand, chemical methods are cheaper and much more easier to perform. Photochemical methods are relatively rarely used for the GIC preparation. In this case, intercalate is irradiated by UV light generating free radicals, which oxidize graphite matrix thus promoting the intercalation process. The intercalation of graphite by $\mathrm{FeCl}_{3}$ in chloroform medium can be conducted due to photochemical reactions [14].

GIC with $\mathrm{Re}_{2} \mathrm{O}_{7}$ was synthesized for the first time by Fuzellier [22]. For this preparation, the melted oxide and vapors were used. In both cases, the prepared GICs were characterized by low content of intercalate. In 1991, Scharff et al. have successfully synthesized $\mathrm{Re}_{2} \mathrm{O}_{7}$-GIC by heating the graphite and dirhenium heptoxide in sealed glass ampoules filled with $\mathrm{O}_{2}, \mathrm{~N}_{2}$, or $\operatorname{Ar}$ [18]. They obtained $\mathrm{Re}_{2} \mathrm{O}_{7}$-GIC with mixed third and fourth stages. Fröba et al. investigated the structure of $\mathrm{Re}_{2} \mathrm{O}_{7}$-GIC using X-ray adsorption near-edge structure $[23,24]$. They noted that charge transfer from graphite to rhenium occurs and between the graphene layers slightly disordered tetrahedral symmetry of $\mathrm{ReO}_{4}{ }^{-}$ion appears. Electrochemical method of $\mathrm{HReO}_{4}$-GIC formation was studied also by Scharff [25]. An anodic oxidation of highly oriented pyrolytic graphite (HOPG) in $65-70 \% \mathrm{HReO}_{4}$ by voltammetry technique yielded 3-stage GIC with a ninterlayer distance of $1370 \mathrm{pm}$.

Due to redox character of rhenium oxides, they can be applied in different types of catalytic reactions. For example, $\mathrm{Re}_{2} \mathrm{O}_{7}$-based catalysts reveal high catalytic activity in olefin metathesis with high efficiency at room temperature [26, 27]. Additionally, catalysts containing $\mathrm{ReO}_{\mathrm{x}}$ demonstrate high catalytic selectivity and activity for partial oxidation of methanol to methylal [28-30].

Exposition of GIC to a high temperature causes evaporation and thermal decomposition of intercalate occupied the interlayer spaces of graphite matrix. Accumulation of gases and increasing pressure lead to the damage of graphene layers. Due to the rapid removal of intercalate, the graphite structure expands up to hundreds of times along the crystallographic $c$ axis yielding exfoliated graphite (EG) [31, 32]. Such a material is characterized by low density, relatively high specific area, hydrophobicity, and high thermal stability [33-35]. Generally, the properties of obtained EG depend on the type of GIC used for synthesis as well as conditions employed during exfoliation process. EG finds application as a precursor for flexible graphite foil preparation, filler in composites, material for oil sorption, or as a matrix for composite preparation $[32,33,35-38]$.

The thermal exfoliation of GIC containing intercalates with catalytic activity can lead to transfer of intercalate from GIC matrix to developed surface area of EG yielding EG/catalyst system. Such composites can be used in chemical power sources production as active material for hydrogen storage or methanol electrooxidation.
In this work, we present the preparation of EG-rhenium oxide composites through shock thermal exfoliation of the beforehand synthesized $\mathrm{HReO}_{4}$-GIC. GIC with perrhenic acid was prepared by anodic oxidation of natural graphite in 75 $80 \% \mathrm{HReO}_{4}$ electrolyte using galvanostatic technique. The synthesized graphitic materials were thermally treated in $\mathrm{N}_{2}$ atmosphere in $700{ }^{\circ} \mathrm{C}$ to conduct exfoliation. Structural changes of the gathered samples were investigated by XRD and Raman spectroscopy, whereas morphology was examined by SEM analysis. Surface composition of the synthesized composites was studied using an energy-dispersive X-ray spectroscopy (EDS). The characterization of as prepared materials was completed be the calculation of the BrunauerEmmet-Teller (BET) surface area.

\section{Experimental}

\section{Sample preparation}

Natural graphite flakes $(170-283 \mu \mathrm{m}$, purity $99.5 \%$, Graphit Kropfmühl) were anodically oxidized using galvanostatic technique and current density equal to $40 \mathrm{~mA}$ $\mathrm{g}^{-1}$. Graphite $(400 \mathrm{mg})$ was closed in current collector made of platinum gauze (purity 99.9\% 120 mesh, Goodfellow). A counter electrode made of platinum wire (purity $99.9 \%, 1 \mathrm{~mm}$ diameter, Goodfellow) was used. Both electrodes were immersed in a threeelectrode reactor beforehand filled with $\mathrm{HReO}_{4}$ solution (75-80\%, Sigma-Aldrich). Anodic and cathodic chambers were separated by glass frit. As a reference electrode, the $\mathrm{Hg} / \mathrm{Hg}_{2} \mathrm{SO}_{4} / 1 \mathrm{M} \mathrm{H}_{2} \mathrm{SO}_{4}$ was used, which was connected with working electrode by salt bridge filled with $1 \mathrm{M} \mathrm{H}_{2} \mathrm{SO}_{4}$. Different charge densities ranging from 60 to $1800{ }^{\circ} \mathrm{C} \mathrm{g}^{-1}$ were passed through the working electrode yielding samples denoted as ReGIC60, ReGIC120, ReGIC600, and ReGIC1800. After, electrochemical process samples were washed with acetone to prevent the deintercalation of perrhenic acid.

EG- $\mathrm{ReO}_{2} / \mathrm{ReO}_{3}$ composites were synthesized using shock thermal treatment under a constant flow of $\mathrm{N}_{2}$. For this purpose, quartz crucible filled with GIC was placed in half of quartz tube which was outside the heating zone of the tube furnace. When the temperature of $700{ }^{\circ} \mathrm{C}$ was reached, GIC was rapidly moved to the heating zone causing the simultaneous exfoliation and deposition of rhenium oxides on graphene layers surface. After $4 \mathrm{~min}$, the composite was moved out of the heating zone to cool down until room temperature was reached. Depending on the exfoliated material, obtained materials were named as Ex-ReGIC60, Ex-ReGIC120, ExReGIC600, and Ex-ReGIC1800. 


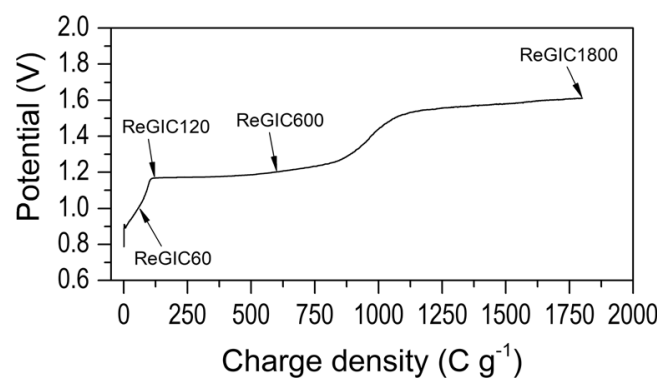

Fig. 1 Galvanostatic curve recorded during electrochemical oxidation of graphite in $75-80 \%$ perrhenic acid

\section{Instrumentation}

All electrochemical measurements were performed using potentiostat/galvanostat Autolab PGSTAT 302N. Raman spectra were recorded by inVia Renishaw micro-Raman system with Ar laser and wavelength $514.5 \mathrm{~nm}$. XRD diffractograms were obtained using PANalytical diffractometer with $\mathrm{Cu} \mathrm{K} \alpha$ radiation $(0.154 \mathrm{~nm})$ with step size of $0.04^{\circ}$ and range from 4 to $60^{\circ}$. Morphology and chemical surface composition of synthesized carbon materials were investigated by SEM equipped with EDS detector using S-3400N Hitachi microscope. Specific surface area of exfoliated samples was gained from $\mathrm{N}_{2}$ adsorption isotherms measured at $77 \mathrm{~K}$ with 3Flex Micromeritics apparatus.

\section{Results and discussion}

\section{Electrochemical oxidation of graphite in $\mathbf{7 5 - 8 0 \%}$ $\mathrm{HReO}_{4}$}

Galvanostatic curve recorded during anodic oxidation of graphite in perrhenic acid solution is presented in Fig. 1. The potential jump to $0.9 \mathrm{~V}$ is associated with the formation of electrical double layer. Next, the gentle potential increment to around $1.15 \mathrm{~V}$ depicts the formation of $\mathrm{HReO}_{4}$-GIC with higher stages. It cannot be excluded that in parallel the electrochemical deposition of

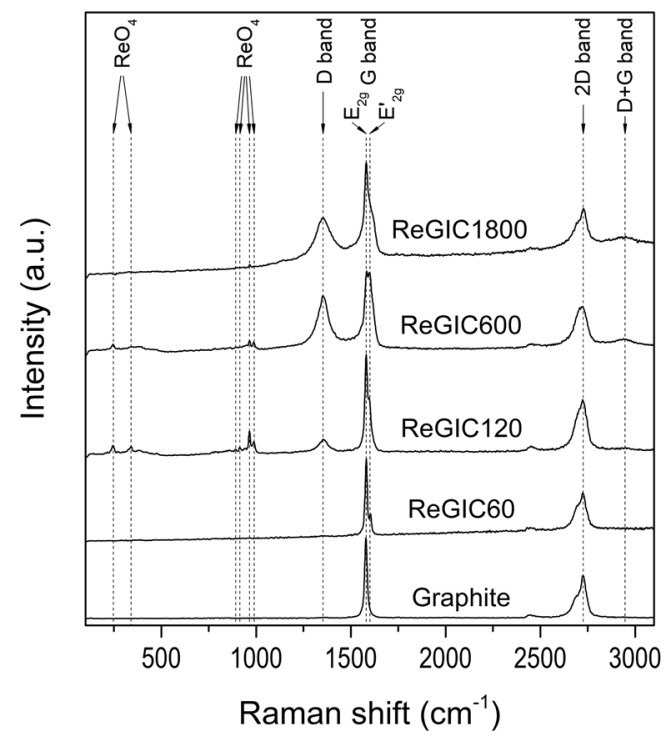

Fig. 3 Raman spectra recorded for pristine graphite and $\mathrm{HReO}_{4}$-GICs

perrhenic acid in form of $\mathrm{H}\left(\mathrm{ReO}_{4}\right)\left(\mathrm{H}_{2} \mathrm{O}\right)$ may also occurs [39]. Potential plateau ranged between the charge densities from 120 to $900{ }^{\circ} \mathrm{C} \mathrm{g}^{-1}$ is connected to further deposition of $\mathrm{HReO}_{4}$ on graphite surface as well as filling the interlayer spaces between the graphene layers. The last potential plateau starting at around $1.5 \mathrm{~V}$ corresponds to the partial oxidation of graphene layers and water electrolysis leading to deintercalation of $\mathrm{HReO}_{4}$. Measured weights of ReGIC60, ReGIC120, ReGIC600, and ReGIC1800 were equal to $517,606,803$, and $659 \mathrm{mg}$, respectively.

From SEM images (Fig. 2) of graphite electrooxidized in $\mathrm{HReO}_{4}$ solution, it can be noted that during the anodic treatment the deposition of $\mathrm{HReO}_{4}$ forming $\mathrm{H}\left(\mathrm{ReO}_{4}\right)\left(\mathrm{H}_{2} \mathrm{O}\right)$ on graphite surface and edges occurs [39]. The amount as well as size of the deposited $\mathrm{H}\left(\mathrm{ReO}_{4}\right)\left(\mathrm{H}_{2} \mathrm{O}\right)$ increases with the treatment time. For the sample ReGIC1800 (Fig. 2 g, h), the insignificant electrochemical exfoliation of graphite flakes can be observed likely caused by the partial oxidation of graphene layers as well as electrolysis of intercalates closed between the graphene layers.

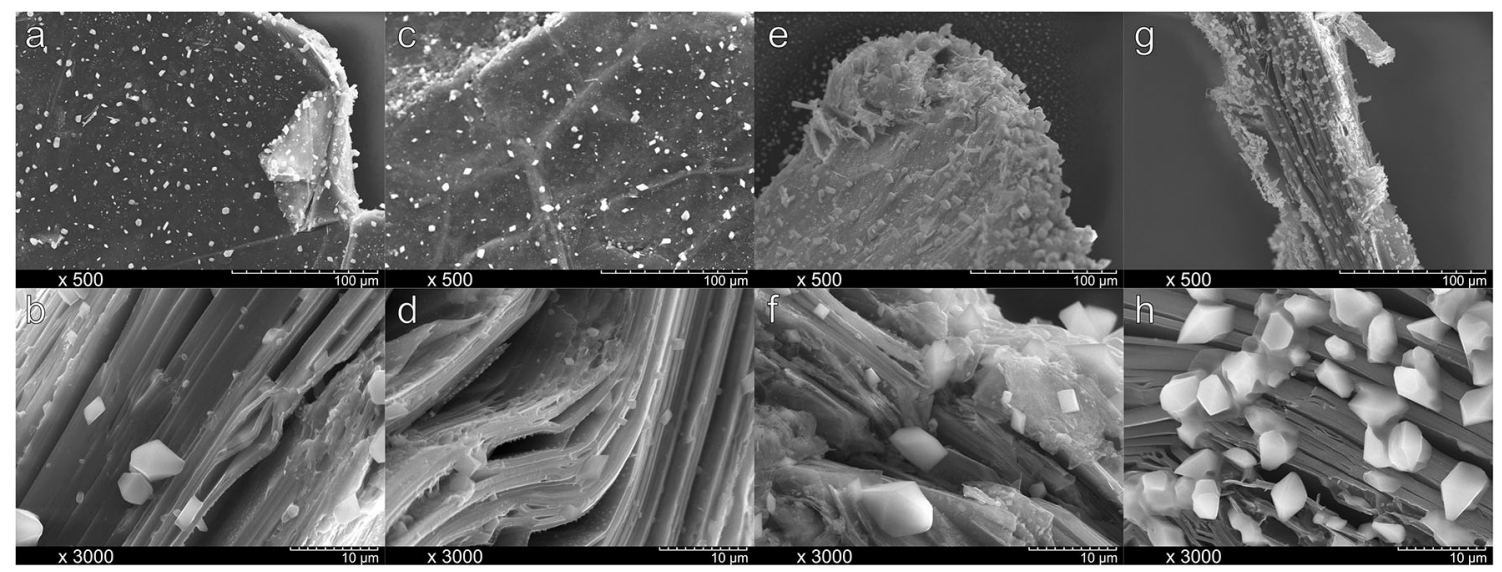

Fig. 2 SEM images of ReGIC60 (a, b), ReGIC120 (c, d), ReGIC600 (e, f), and ReGIC1800 (g, h) 


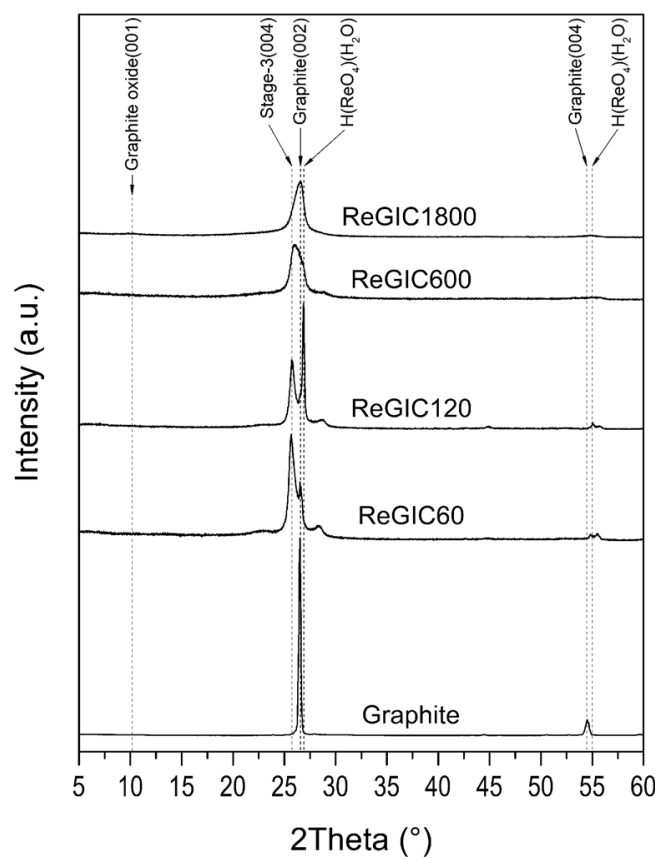

Fig. 4 XRD patterns recorded for pristine graphite and $\mathrm{HReO}_{4}$-GICs

Raman spectra recorded for graphite and synthesized GICs are shown in Fig. 3. Typical bands for carbon materials are observed at $1355,1580,2725$, and $2910 \mathrm{~cm}^{-1}$. The first one, D band, is associated with the presence of $\mathrm{sp}^{3}$ hybridized carbon deriving from defects, edges, or functional groups [40, 41]. It can be noted that elongation of graphite oxidation causes the increase of $\mathrm{D}$ band intensity, due to the formation of oxygen functionalities on the surface of graphene layers. The second one, called as $\mathrm{G}$ band, is assigned to the first-order scattering of $\mathrm{E}_{2 \mathrm{~g}}$ mode arising from the $\mathrm{sp}^{2}$ bonded carbon [40, 42]. For graphite, the regarded band has one maximum, whereas Raman spectra for $\mathrm{HReO}_{4}$-GICs reveal the second maximum of $\mathrm{G}$ band, called $\mathrm{E}_{2 \mathrm{~g}}^{\prime}$. It evidences the presence of intercalated graphite structure. During the anodic treatment of graphite, the intensity of $E_{2 g}^{\prime}$ band increases compared with $E_{2 g}$ band thus indicating the enhanced amount of intercalated $\mathrm{HReO}_{4}[43$,
44]. Surprisingly, in the case of sample ReGIC1800, the intensity of $E_{2 \mathrm{~g}}^{\prime}$ band in turn decreases. Such a behavior is caused by the partial degradation of graphitic structure due to oxidation of graphene layers accompanied by the partial deintercalation of $\mathrm{ReO}_{4}{ }^{-}$ions from graphite matrix. 2D band comes from the splitting of $\pi$ electron dispersion energies and consists of two peaks [40-42]. The last band seen in Fig. 3, called as $\mathrm{D}+\mathrm{G}$ band, is characteristic for oxidized graphene layers $[45,46]$. For graphite and ReGIC60, the abovementioned band is absent. It appears only on Raman spectra for samples ReGIC120, ReGIC600, and ReGIC1800. Its intensity increases with time of electrochemical oxidation. Such a behavior coincides with electrochemical results revealing that prolongation of anodic treatment of graphite leads to the partial oxidation of graphite matrix. Raman bands recorded under $1000 \mathrm{~cm}^{-1}$ can be assigned to the appearance of tetrahedral $\mathrm{ReO}_{4}{ }^{-}$groups [47-50]. The low-frequency modes at 245 and $341 \mathrm{~cm}^{-1}$ correspond to the bending motion of Re-O bonds $[50,51]$, whereas modes with higher frequencies at $889,914,965$, and $988 \mathrm{~cm}^{-1}$ arise from the stretching vibration of $\mathrm{Re}-\mathrm{O}$ bonds in $\mathrm{ReO}_{4}{ }^{-}$tetrahedra [48, 50, 51]. The most pronounced effect of $\mathrm{ReO}_{4}{ }^{-}$bands is present for sample ReGIC120. Further, electrooxidation causes the diminution in their intensities, which indicate that deintercalation of perhenic ions as well as deposition of $\mathrm{H}\left(\mathrm{ReO}_{4}\right)\left(\mathrm{H}_{2} \mathrm{O}\right)$ on graphite surface takes place.

XRD measurements for graphite and oxidized samples provided information on changes in structure caused by electrochemical treatment of a graphite in perrhenic acid (Fig. 4). XRD pattern of graphite shows two main diffraction peaks at 26.6 and $54.4^{\circ}$ revealing that the interlayer distance between the graphene layers is equal to $3.35 \AA$ [52]. For sample ReGIC60, a new diffraction peak at $25.7^{\circ}$ corresponding to stage-3 $\mathrm{HReO}_{4}$-GIC with average interlayer distance of 13.8 $\AA$ can be observed. This result is in agreement with the results presented by Scharff $[18,25]$. XRD spectrum of graphite being oxidized using charge density of $120^{\circ} \mathrm{C} \mathrm{g}^{-1}$ involves two

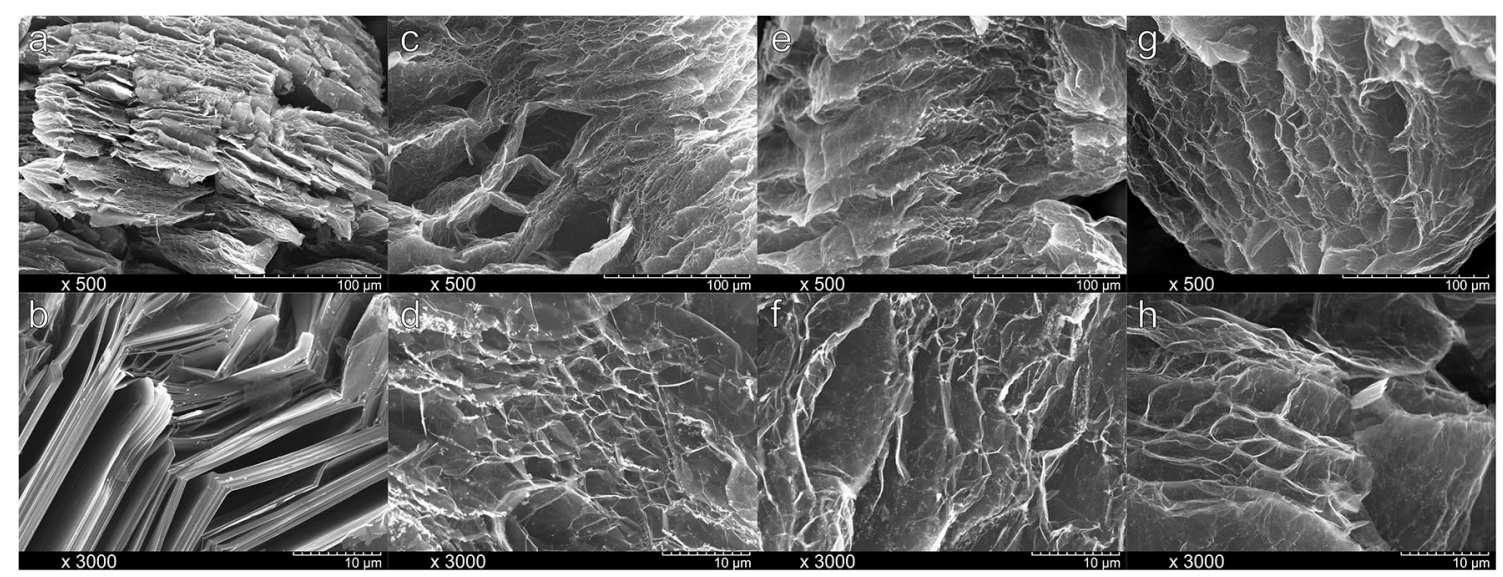

Fig. 5 SEM images of Ex-ReGIC60 (a, b), Ex-ReGIC120 (c, d), Ex-ReGIC600 (e, f) and Ex-ReGIC1800 (g, h) 


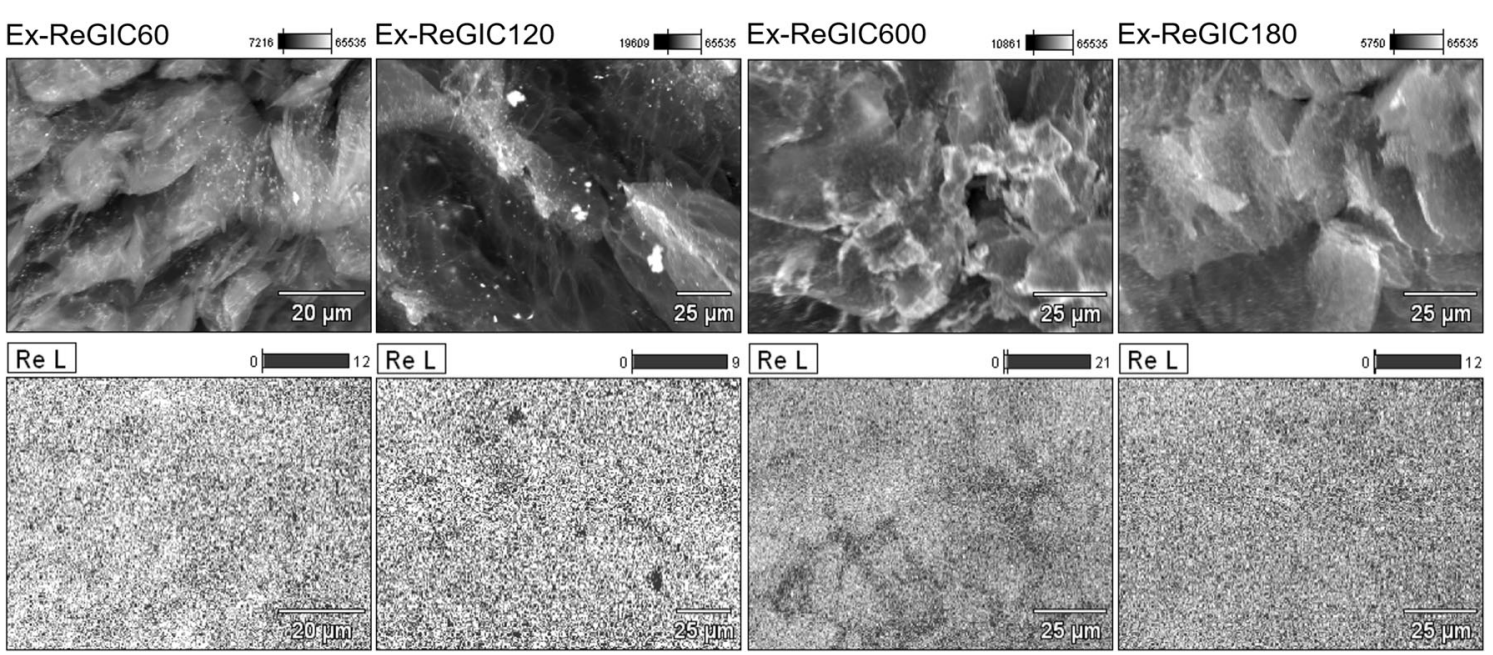

Fig. 6 SEM and EDS maps of exfoliated graphite-rhenium oxide composites

sharp diffraction peaks at 26.9 and $55.0^{\circ}$. Those peaks confirm the formation of $\mathrm{H}\left(\mathrm{ReO}_{4}\right)\left(\mathrm{H}_{2} \mathrm{O}\right)$ particles on the graphite surface. Further oxidation of graphite in $\mathrm{HReO}_{4}$ solution leads to the degradation of stage-3 GIC and partial oxidation of graphite matrix. For ReGIC1800, an insignificant peak at $10.2^{\circ}$ can be observed. It likely means the existence of graphite oxide structure within the deeply oxidized sample [53, 54].

\section{Thermal exfoliation of the previously synthesized $\mathrm{HReO}_{4}$-GIC}

The morphological changes in GIC structure caused by thermal treatment under ambient atmosphere were investigated using SEM (Fig. 5). As one can see, the shock treatment of GICs performed in $\mathrm{N}_{2}$ atmosphere contributed to the deformation and separation of graphene layers. With increasing intercalation duration, an exfoliation process becomes more efficient. This correlation is clearly connected with the increasing amount of perrhenic acid ions between the graphene layers of exfoliated GIC. Partial transformation of graphite to graphite oxide during the electrochemical overoxidation may be also responsible for the above-mentioned tendency. From the presented SEM images, it can be also noted that all exfoliated materials are covered by rhenium oxides particles. BET measurements reveal that samples Ex-ReGIC60, ExReGIC120, Ex-ReGIC600, and Ex-ReGIC1800 have the surface areas equal to $3.5,6.2,18.5$, and $19.6 \mathrm{~m}^{2} \mathrm{~g}^{-1}$, respectively. EDS mapping shows homogeneous distribution of rhenium oxides over the exfoliated materials (Fig. 6). The highest atomic percent concentration of rhenium in reached for sample Ex-GIC600 (Table 1). Decrease in Re amount in sample Ex-GIC1800 is likely caused by the partial deintercalation during overoxidation of GIC.

Raman spectra of exfoliated samples are depicted in Fig. 7. As one can see, due to thermal treatment, the intensity of $\mathrm{D}$ band decreased noticeably as compared with $\mathrm{HReO}_{4}$ GIC samples (Fig. 3). It means that thermal treatment under nitrogen atmosphere contributes to the structure ordering. The only sample that exhibits the significant amount of surface defect is Ex-ReGIC1800. In this case, lack of structural ordering likely results the partial electrochemical exfoliation that probably occurs during the overoxidation of graphite in perrhenic acid over potential $1.5 \mathrm{~V}$. It cannot be also excluded that violent removal of oxygen functionalities which formed during overoxidation of graphite generated higher amounts of defects as compared with other exfoliated samples. The presence of oxygen functionalities on the surface of graphene layer of Ex-ReGIC1800 is also confirmed by weak $\mathrm{D}+\mathrm{G}$ band at $2910 \mathrm{~cm}^{-1}$ (Fig. 7). After the thermal treatment of $\mathrm{HReO}_{4}$-GICs, Raman spectra do not involve bands corresponding to tetrahedral $\mathrm{ReO}_{4}{ }^{-}$. On the other hand, new bands at 141, 166, 198, and 370 $\mathrm{cm}^{-1}$ appeared on the regarded spectra. Bands with maximum at 198 and $370 \mathrm{~cm}^{-1}$ are attributed to $\mathrm{ReO}_{3}$ structure [48, 55]. Other two bands at 141 and $166 \mathrm{~cm}^{-1}$ can be assigned to $\mathrm{O}=\mathrm{Re}=\mathrm{O}$ bonds deriving from $\mathrm{ReO}_{2}$.
Table 1 Atomic \% content of exfoliated graphite-rhenium oxide composites

\begin{tabular}{lllll}
\hline Sample & Ex-ReGIC60 & Ex-ReGIC120 & Ex-ReGIC600 & Ex-ReGIC1800 \\
\hline C atom \%: & 96.31 & 94.92 & 85.59 & 89.96 \\
O atom \%: & 3.47 & 4.75 & 12.14 & 8.95 \\
Re atom \%: & 0.22 & 0.33 & 2.27 & 1.09 \\
\hline
\end{tabular}




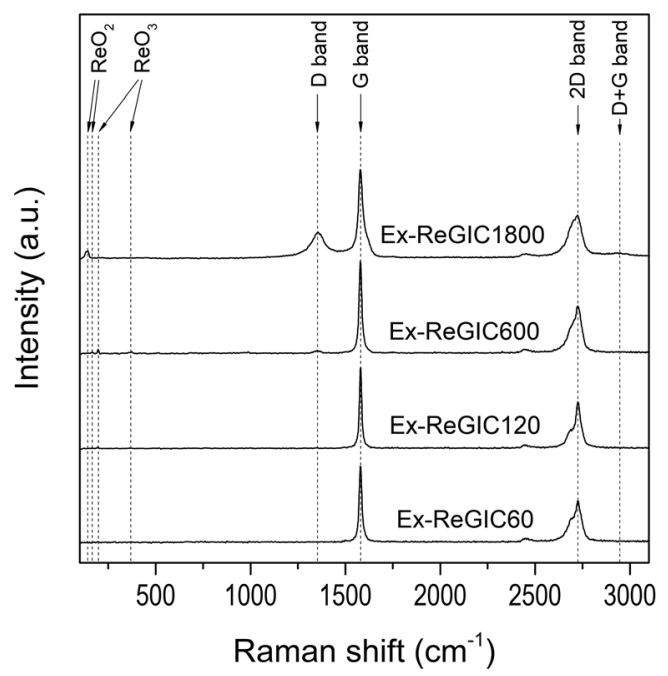

Fig. 7 Raman spectra recorded for exfoliated graphite-rhenium oxide composites

XRD patterns of thermally EG-rhenium oxide composites are presented in Fig. 8. Due to insignificant amount of intercalate within the ReGIC60 sample, most of graphitic structure is preserved after the thermal treatment process. It is manifested by the presence of sharp peak at $26.6^{\circ}$. On the other hand, relatively weak peaks arising with the appearance cubic $\mathrm{ReO}_{3}$ and rhombohedral $\mathrm{ReO}_{2}$ are also present $[56,57]$. Diffraction patterns of Ex-ReGIC120, Ex-ReGIC600, and Ex-ReGIC1800 show that with increasing charge density consumed to anodic oxidation of graphite, the intensity of peaks deriving from the rhenium oxides also increases. It is worth

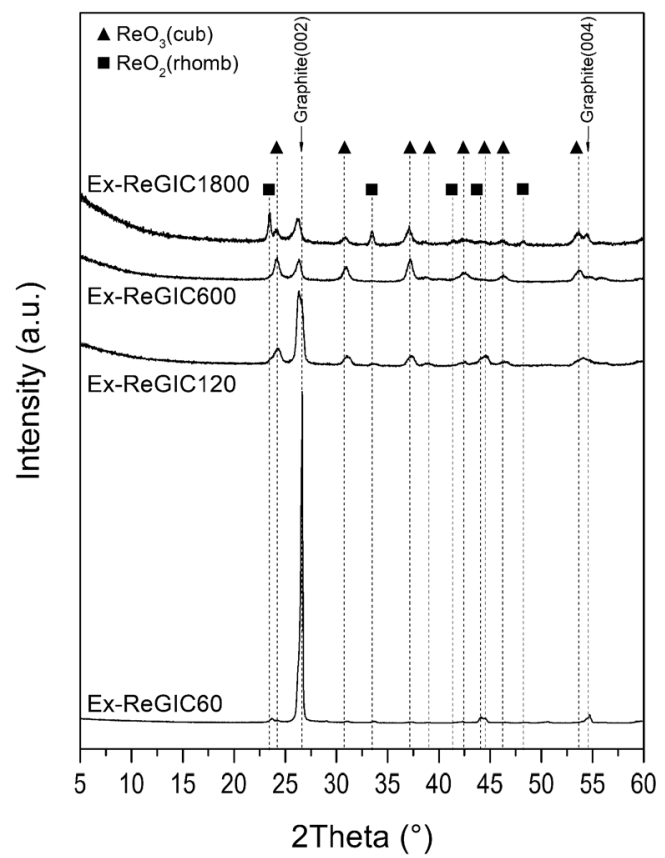

Fig. 8 XRD patterns recorded for exfoliated graphite-rhenium oxide composites to note that Ex-ReGIC600 sample is composed mostly of $\mathrm{EG}$ and $\mathrm{ReO}_{3}$, whereas the graphene layers in the case of sample Ex-ReGIC1800 are covered by $\mathrm{ReO}_{3}$ and $\mathrm{ReO}_{2}$. It means that thermal exfoliation of sample ReGIC1800 with lower concentration of perrhenic acid promotes the $\mathrm{ReO}_{2}$ formation, whereas thermal treatment of ReGIC600 with higher amount of $\mathrm{HReO}_{4}$ leads to the formation of $\mathrm{ReO}_{3}$.

\section{Conclusions}

In our work, we describe the two-step process of EG-rhenium oxide composite preparation. Electrochemical oxidation of graphite in aqueous solution of $\mathrm{HReO}_{4}$ leads to intercalation of $\mathrm{ReO}_{4}{ }^{-}$ion between the interlayer spaces thus yielding of stage-3 GIC. Simultaneously, perrhenic acid is electrodeposited on graphite surface and edges forming $\mathrm{H}\left(\mathrm{ReO}_{4}\right)\left(\mathrm{H}_{2} \mathrm{O}\right)$. Due to long-term oxidation, graphite is partially exfoliated and deintercalation of $\mathrm{ReO}_{4}{ }^{-}$occurs. Thermal treatment in nitrogen atmosphere of oxidized graphite in perrhenic acid causes the separation of graphene layers and simultaneous deposition of rhenium oxides of it surface. The synthesized EGs are characterized by developed specific surface area up to $19 \mathrm{~m}^{2} \mathrm{~g}^{-1}$. Taking into account the rhenium oxides on the surface of EG compounds, it should be emphasized that to their formation the deposition of perrhenic acid during electrochemical oxidation desired. XRD patterns of the synthesized composites reveal that thermal treatment of $\mathrm{HReO}_{4^{-}}$ GICs leads to the formation of cubic $\mathrm{ReO}_{3}$ and rhombohedral $\mathrm{ReO}_{2}$. The synthesized EG-rhenium oxide composites can be potentially used as a catalysts for methanol oxidation to methylal.

Funding information This research was financially supported by the National Science Centre, Poland (2018/02/X/ST5/02765). This research was financially supported by the Ministry of Science and Higher Education.

\section{Compliance with ethical standards}

Conflict of interest The authors declare that they have no conflict of interest.

Open Access This article is licensed under a Creative Commons Attribution 4.0 International License, which permits use, sharing, adaptation, distribution and reproduction in any medium or format, as long as you give appropriate credit to the original author(s) and the source, provide a link to the Creative Commons licence, and indicate if changes were made. The images or other third party material in this article are included in the article's Creative Commons licence, unless indicated otherwise in a credit line to the material. If material is not included in the article's Creative Commons licence and your intended use is not permitted by statutory regulation or exceeds the permitted use, you will need to obtain permission directly from the copyright holder. To view a copy of this licence, visit http://creativecommons.org/licenses/by/4.0/. 


\section{References}

1. Chung DDL (2002) Review: graphite. J Mater Sci 37(8):14751489

2. Forsman WC, Dziemianowicz T, Leong K, Carl D (1983) Graphite intercalation chemistry: an interpretive review. Synth Met 5(2):77100

3. Zabel H, Solin S (1990) Graphite intercalation compounds I. Springer, Berlin Heidelberg, Berlin, Heidelberg

4. Besenhard JO (1976) The electrochemical preparation and properties of ionic alkali metal-and NR4-graphite intercalation compounds in organic electrolytes. Carbon 14(2):111-115

5. Besenhard JO, Minderer P, Bindl M (1989) Hydrolysis of perchloric acid and sulfuric acid graphite intercalation compounds. Synth Met 34(1-3):133-138

6. Scharff P, Xu ZY, Stumpp E, Barteczko K (1991) Reversibility of the intercalation of nitric acid into graphite. Carbon 29(1):31-37

7. Rozmanowski T, Krawczyk P (2019) Electrocatalytic properties of graphite intercalation compound with metal chlorides modified by cathodic treatment. Electrochim Acta 297:735-741

8. Matsumoto R, Okabe Y (2016) Electrical conductivity and air stability of $\mathrm{FeCl} 3, \mathrm{CuCl} 2, \mathrm{MoCl} 5$, and $\mathrm{SbCl} 5$ graphite intercalation compounds prepared from flexible graphite sheets. Synth Met 212: 62-68

9. Inagaki M, Iwashita N, Kouno E (1990) Potential change with intercalation of sulfuric acid into graphite by chemical oxidation. Carbon 28(1):49-55

10. Tryba B, Przepiórski J, Morawski AW (2003) Influence of chemically prepared $\mathrm{H} 2 \mathrm{SO} 4$-graphite intercalation compound (GIC) precursor on parameters of exfoliated graphite (EG) for oil sorption from water. Carbon 41(10):2013-2016

11. Krawczyk P, Gurzęda B (2016) Electrochemical properties of exfoliated graphite affected by its two-step modification. J Solid State Electrochem 20(2):361-369

12. Zhao J, Zou X, Zhu Y, Xu Y, Wang C (2016) Electrochemical intercalation of potassium into graphite. Adv Funct Mater 26(44): 8103-8110

13. Xu J, Dou Y, Wei Z et al (2017) Recent progress in graphite intercalation compounds for rechargeable metal (Li, Na, K, Al)-ion batteries. Adv Sci 4:1700146

14. Schlögl R, Boehm HP (1988) Photochemical intercalation in graphite. Synth Met 23(1-4):407-413

15. Lee BJ (2002) Characteristics of exfoliated graphite prepared by intercalation of gaseous SO3 into graphite. Bull Korean Chem Soc 23:1801-1805

16. Fuzellier H, Lelaurain M, Marêché JF (1989) The graphite nitrate compounds: Graphite-N2O5 system. Synth Met 34(1-3):115-120

17. Tongay S, Hwang J, Tanner DB, Pal HK, Maslov D, Hebard AF (2010) Supermetallic conductivity in bromine-intercalated graphite. Phys Rev B - Condens Matter Mater Phys 81(11):115428

18. Scharff P, Stumpp E, Höhne M, Wang YX (1991) Upon the intercalation of rhenium heptoxide and rhenium trioxide nitrate into graphite. Carbon 29(4-5):595-597

19. Ren H, Kang FY, Jiao QJ, Shen WC (2009) Synthesis of a metal chloride-graphite intercalation compound by a molten salt method. New Carbon Mater 24(1):18-22

20. Rozmanowski T, Krawczyk P (2018) Influence of chemical exfoliation process on the activity of $\mathrm{NiCl} 2-\mathrm{FeCl} 3-\mathrm{PdCl} 2$-graphite intercalation compound towards methanol electrooxidation. Appl Catal B Environ 224:53-59

21. Gurzeda B, Krawczyk P (2019) Electrochemical formation of graphite oxide from the mixture composed of sulfuric and nitric acids. Electrochim Acta 310:96-103

22. Fuzellier H (1974) PhD Thesis, University of Nancy, France
23. Fröba M, Lochte K, Metz W (1994) XAS and XRD Studies on graphite intercalation compounds of Re2O7. Mol Cryst Liq Cryst Sci Technol Sect A Mol Cryst Liq Cryst 244(1):239-244

24. Fröba M, Metz W (1997) Phase transitions at room temperature in graphite intercalation compounds of Re2O7. Solid State Ionics 101-103:619-623

25. Scharff P (1989) Elektrochemische Untersuchungen an Graphitsalzen mit $\mathrm{HNO} 3, \mathrm{HClO} 4, \mathrm{HReO} 4$ und halogenierten Essigsäuren / electrochemical investigations on graphite salts with $\mathrm{HNO} 3, \mathrm{HClO} 4, \mathrm{HReO} 4$ and halogenated acetic acids. Zeitschrift für Naturforsch B 44(7):772-777

26. Mol JC (1999) Olefin metathesis over supported rhenium oxide catalysts. Catal. Today 51(2):289-299

27. Oikawa T, Ookoshi T, Tanaka T, Yamamoto T, Onaka M (2004) A new heterogeneous olefin metathesis catalyst composed of rhenium oxide and mesoporous alumina. Microporous Mesoporous Mater 74(1-3):93-103

28. Nikonova OA, Capron M, Fang G, Faye J, Mamede AS, Jalowiecki-Duhamel L, Dumeignil F, Seisenbaeva GA (2011) Novel approach to rhenium oxide catalysts for selective oxidation of methanol to DMM. J Catal 279(2):310-318

29. Chan ASY, Chen W, Wang H, Rowe JE, Madey TE (2004) Methanol reactions over oxygen-modified re surfaces: influence of surface structure and oxidation. J. Phys. Chem. B 108(38): 14643-14651

30. Yuan Y, Shido T, Iwasawa Y (2000) The new catalytic property of supported rhenium oxides for selective oxidation of methanol to methylal. Chem Commun 1421-1422

31. Anderson SH, Chung DDL (1984) Exfoliation of intercalated graphite. Carbon 22(3):253-263

32. Chung DDL (1987) Exfoliation of graphite. J Mater Sci 22(12): 4190-4198

33. Yakovlev AV, Finaenov AI, Zabud'kov SL, Yakovleva EV (2006) Thermally expanded graphite: synthesis, properties, and prospects for use. Russ J Appl Chem 79(11):1741-1751

34. Skowroński JM, Krawczyk P, Rozmanowski T, Urbaniak J (2008) Electrochemical behavior of exfoliated NiCl2-graphite intercalation compound affected by hydrogen sorption. Energy Convers Manag 49(9):2440-2446

35. Bian J, Xiao M, Wang SJ, Lu YX, Meng YZ (2009) Novel application of thermally expanded graphite as the support of catalysts for direct synthesis of DMC from $\mathrm{CH} 3 \mathrm{OH}$ and $\mathrm{CO} 2$. J Colloid Interface Sci 334(1):50-57

36. Yao T, Zhang Y, Xiao Y, Zhao P, Guo L, Yang H, Li F (2016) The effect of environmental factors on the adsorption of lubricating oil onto expanded graphite. J Mol Liq 218:611-614

37. Zheng G, Wu J, Wang W, Pan C (2004) Characterizations of expanded graphite/polymer composites prepared by in situ polymerization. Carbon 42(14):2839-2847

38. Krawczyk P, Rozmanowski T, Osińska M (2016) Electrochemical sorption of hydrogen in exfoliated graphite/nickel/palladium composite. Int J Hydrogen Energy 41(45):20433-20438

39. Vargas-Uscategui A, Mosquera E, López-Encarnación JM, Chornik B, Katiyar RS, Cifuentes L (2014) Characterization of rhenium compounds obtained by electrochemical synthesis after aging process. J Solid State Chem 220:17-21

40. Ferrari AC (2007) Raman spectroscopy of graphene and graphite: disorder, electron-phonon coupling, doping and nonadiabatic effects. Solid State Commun 143(1-2):47-57

41. Pimenta MA, Dresselhaus G, Dresselhaus MS, Cançado LG, Jorio A, Saito R (2007) Studying disorder in graphite-based systems by Raman spectroscopy. Phys Chem Chem Phys 9(11):1276-1291

42. Malard LM, Pimenta MA, Dresselhaus G, Dresselhaus MS (2009) Raman spectroscopy in graphene. Phys Rep 473(5-6):51-87 
43. Alsmeyer DC, McCreery RL (1992) In situ raman monitoring of electrochemical graphite intercalation and lattice damage in mild aqueous acids. Anal Chem 64(14):1528-1533

44. Chacón-Torres JC, Wirtz L, Pichler T (2014) Raman spectroscopy of graphite intercalation compounds: charge transfer, strain, and electron-phonon coupling in graphene layers. Phys Status Solidi 251(12):2337-2355

45. Kudin KN, Ozbas B, Schniepp HC, Prud'homme RK, Aksay IA, Car R (2008) Raman spectra of graphite oxide and functionalized graphene sheets. Nano Lett 8(1):36-41

46. Dimiev AM, Tour JM (2014) Mechanism of graphene oxide formation. ACS Nano 8(3):3060-3068

47. Thevenet A, Marie C, Tamain C, Amendola V, Miljkovic A, Guillaumont D, Boubals N, Guilbaud P (2020) Perrhenate and pertechnetate complexation by an azacryptand in nitric acid medium. Dalt Trans 49(5):1446-1455

48. Andriopoulou C, Anastasiou I, Boghosian S (2019) Di-oxo and trioxo Re(VII)-oxosulfato complexes in the Re2O7-K2S2O7 molten system. Molecular structure, vibrational properties and temperature-dependent interconversion. Vib Spectrosc 100:14-21

49. Lacheen HS, Cordeiro PJ, Iglesia E (2007) Isolation of rhenium and $\mathrm{ReOx}$ species within ZSM5 channels and their catalytic function in the activation of alkanes and alkanols. Chem - A Eur J 13(11): 3048-3057
50. Purans J, Kuzmin A, Cazzanelli E, Mariotto G (2007) Disorderinduced Raman scattering in rhenium trioxide (ReO3). J Phys Condens Matter 19(22):226206-226214

51. Kon Y, Araque M, Nakashima T, Paul S, Dumeignil F, Katryniok B (2017) Direct Conversion of glycerol to allyl alcohol over aluminasupported rhenium oxide. ChemistrySelect 2(30):9864-9868

52. Li ZQ, Lu CJ, Xia ZP, Zhou Y, Luo Z (2007) X-ray diffraction patterns of graphite and turbostratic carbon. Carbon 45(8):16861695

53. Nakajima T, Matsuo Y (1994) Formation process and structure of graphite oxide. Carbon 32(3):469-475

54. Krishnamoorthy K, Veerapandian M, Yun K, Kim SJ (2013) The chemical and structural analysis of graphene oxide with different degrees of oxidation. Carbon 53:38-49

55. Beattie IR, Ozin GA (1969) Vibrational spectrum of gaseous, liquid, and solid Re2O7. J Chem Soc A Inorganic, Phys Theor Chem: 2615-2619

56. Biswas K, Muthu DVS, Sood AK, Kruger MB, Chen B, Rao CNR (2007) Pressure-induced phase transitions in nanocrystalline ReO3. J Phys Condens Matter 19(43):436214

57. Shcheglov PA, Drobot DV (2006) Heterogeneous equilibria in the rhenium-oxygen system. Russ J Phys Chem A 80(11):1819-1825

Publisher's note Springer Nature remains neutral with regard to jurisdictional claims in published maps and institutional affiliations. 